\title{
LA INFORMÁTICA Y LAS CIENCIAS NATURALES
}

Hernando González Sierra

Profesor Unviersidad Surcolombiana Neiva,

Programa de Matemáticas y Física

Clotario Israel Peralta Garcia

Profesor Universidad Surcolombiana Neiva,

Programa de Matemáticas y Física.

\section{INTRODUCCIÓN}

El próximo siglo (nuevo milenio), será el siglo de la telemática y de los computadores; las autopistas cibernéticas estarán atiborradas de elementos de información que viajan por el espacio a la velocidad de la luz y su utilización será necesaria en todas las actividades cotidianas. El hombre necesitará aprender cosas mucho más complejas y de manera más rápida, ya no será posible desconocer el papel de la tecnología para posibilitar esta tarea necesaria y

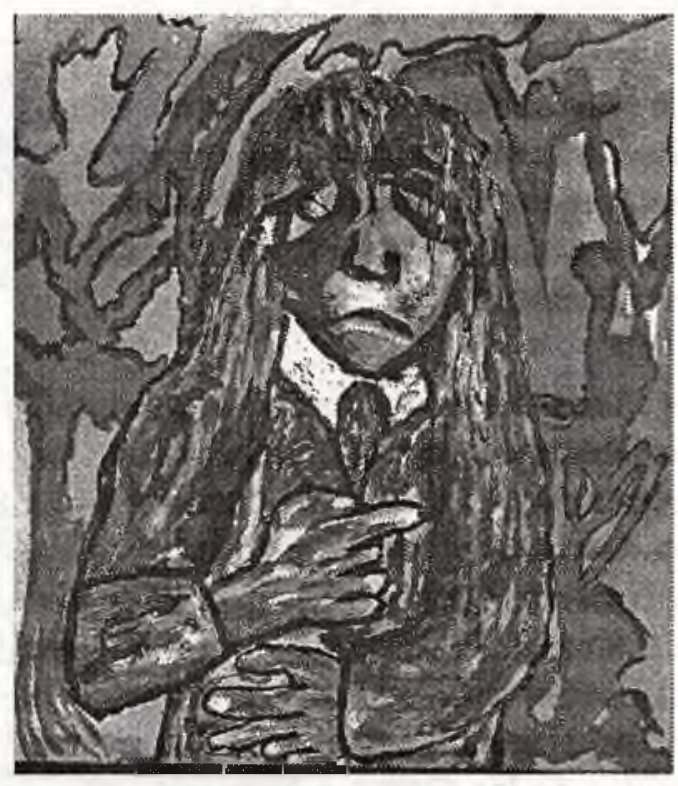
obligatoria para todos. El escenario que tendrán que vivir los niños y jóvenes que hoy asisten a las escuelas, colegios y universidades.

La Universidad Surcolombiana, a través de la Facultad de Educación, que tiene como misión producir y difundir el conocimiento viabilizador del desarrollo humano integral, equitativo y sostenible no puede sustraerse a la tarea de preparar adecuadamente a los educadores para que puedan democratizar el conocimiento y la cultura con calidad y eficiencia.

En este arículo presentamos un resumen de la propuesta de cómo utilizar las herramientas de la Información educativa para constuir un currículo basado en la identificación de problemas y

enmarcado en las nuevas reglamentaciones sobre educación ambiental.

Esta propuesta, pretende abrir un espacio de reflexión y acción para que las ventajas de las nuevas tecnologías faciliten el aprendizaje de las Ciencias Naturales en los niños y jóvenes de la región surcolombiana.

\section{PRESENTACIÓN DEL PROBLEMA}

La última reestructuración del sistema educativo nacional, en los niveles de básica primaria, básica secundaria y media 
vocacional, se llevó a cabo mediante el Decreto Ley No. 088 de 1976, que explica los fines del sistema educativo y señala pautas para el diseño curricular y su administración, y el 1002 de 1984 que establece el plan de estudios vigentes.

En este nuevo marco legal, las ciencias naturales ya no se estudian aisladalmente como disciplinas, sino que han sido integradas bajo la denominación de: CIENCIAS NATURALES INTEGRADA. Sin embargo, éstas se continúan enseñando separadamente a juicio personal, contraviniendo el espíritu de la Ley y sin notarse un progreso significativo en los jóvenes que egresan de las instituciones de enseñanza media vocacional.

La región surcolombiana no cuenta con los docentes preparados para orientar los programas de ciencias naturales Integradas para los niveles de educación básica primaria, básica secundaria y media vocacional de acuerdo con las exigencias del nuevo currículo. Ellos están preparados solamente en la enseñanza de una disciplina particular de las Ciencias Naturales y $P$ en algunos casos excepcionales, son E docentes de otras disciplinas dife- rentes D a su formación profesional, quienes orien- hasta errónea. La construcción del conocimiento con una visión unidisciplinaria presenta la posibilidad de usar vocabulario y terminología no adecuada que distorsiona y dejan parcializada la descripción de los fenómenos que son objeto de estudio.

3. El docente selecciona los temas que supuestamente él domina mejor (los de su especialidad) y elimina en forma arbitraria aquellos que considra irrelevantes o quizás muy complejos (Incompletez). La incompletez en los cursos de Ciencias Naturales priva a mejores educandos, de tópicos interesantes que pueden ser de gran importancia en su futura formación integral.

4. La enseñanza sigue siendo de tipo enciclopédico y memorístico, adoptando los textos que se ofrecen en el mercado sin ningún tipo de adaptación al medio. Los docentes de ciencias naturales de la región surcolombiana, no elaboraron textos adecuados que sean el resultado de su reflexión educativa e innovadora.

Las necesidades presentes en la región surcolombiana, en relación con el personal docente no preparado, permiten asegurar la necesidad de un programa de Especialización en la Docencia de las Ciencias Naturales Integrada. Desde el comienzo del año de 1992, la idea de una Especialización en el área de las Ciencias Naturales, se ha venido madurando con la participación del grupo de docentes del área de Física del programa de Licenciatura en Matemáticas y Física, quienes han manifestado sus inquietudes acerca de la creación e implementación de estudios de Postgrado.

De acuerdo con la Ley General de la Educación (Ley 115 de 1993), las facultades de educación deben ofrecer programas de capacitación, incluido el nivel de Posgrado, a los docentes del sector educativo de 
primaria y secundaria. Además, la ley 30 de 1992 que rige la Educación Superior en general, establece la reglamentación a que se deben someter los programas de especialización de las universidades. En consecuenca las entidades de educación superior tendrán como parte importante de su actividad la investigación, el desarrollo académico y la formación de profesionales a todos los niveles y modalidades de acuerdo a las necesidades y prioridades regionales.

\section{UNA}

ALTERNATIVA DE SOLUCIÓN

La región surcolombiana presenta un considerable retraso en la aplicación de nuevos métodos, técnicas y estrategias para la enseñanza de las Ciencias Naturales en los niveles de: básica primaria, básica secundaria y media vocacional. La capacitación de los docentes, con base en las nuevas herramientas pedagógicas e innovaciones en el avance del conocimiento, es una necesidad urgente que debe implementarse a través de la creación de Postgrados.

La Facultad de Educación de la Universidad Surcolombiana conscienté de la dimensión de este problema educativo y de su condición como centro piloto encargado de propiciar la calidad de la educación a nivel regional. está desarrollando políticas educativas que permitan dar soluciones a mediano y largo plazo.

El currículo vigente para la enseñanza de las Ciencias Naturales en los niveles de: básica primaria, basica secundaria y media vocacional, contempla la Física, la Química, la Biología, la Ecología y Ciencias de la Tierra, ya no como áreas independientes entre si, sino como un área integrada con el nombre de CIENCIAS NATURALES.

Ya esbozados e identificados los problemas en la enseñanza actual de las Ciencias Naturales, hemos elaborado una propuesta curricular que proporcione soluciones a las dificultades ya planteadas: Enseñanza unidisciplinar. incompleta $y$ enciclopédica.

En este orden de ideas, queremos en principio, rescatar el carácter científico que es propio de las Ciencias Naturales, el cual queda desvirtuado al aislar un fenómeno particular de todo su entorno y asociarlo a una sola disciplina, llámese Física, Química, Biología etc. Sabemos que la población a la cual estará dirigida esta Especialización tiene muy buenos conocimientos en su disciplina particular y que ahondar en ella, o en otras que no son de su campo específico de formación en pregrado, no resolvería ninguno de los problemas planteados.

Los conocimientos y las nuevas de la Sicología cognitiva sobre el aprendizaje y 
las características del aprendiz, la emergencia de nuevas aproximaciones epistemológicas sobre la naturaleza de la construcción del conocimiento y las nuevas estrategias instruccionales que de éstas y de aquéllas se derivan tendientes a capacitar al docente para que apoye a sus estudiantes a "aprender a aprender", han generado ya y pueden seguir generando hacia el futuro, proyectos que mejoren los currículos y los métodos de enseñanza de las Ciencias Naturales. Los argumentos sobre el énfasis que se debe dar a los contenidos o a los procesos, podrían diluirse si las nuevas ideas penetran con más fuerza en los centros de formación de maestros y se constituyen en parte esencial bases teóricas para dar lugar a nuevas alternativas de mejoramiento en la enseñanza de las Ciencias Naturales.

Los docentes del área de Física del Programa de Licenciatura en Matemáticas y Física, conocedores de la necesidad de resolver el problema educativo de la enseñanza de las Ciencias Naturales Integradas, han encargado a los profesores de tiempo completo: HERNANDO GONZÁLEZ SIERRA Y CLOTARIO ISRAEL PERALTA GARCIA, la elaboración de una propuesta que de solución a los problemas generados por la implementación de nuevo currículo en la enseñanza de las Ciencias Naturales en la región surcolombiana.

Para tal fin hemos formulado, como alternativa de solución a estas dificultades, la "ESPECIALIZACIÓN EN INFORMATICA PARA LA DOCENCIA DE LAS CIENCIAS NATURALES", que pretende la formación de un grupo de profesionales especializados, en la enseñanza de las Ciencias Naturales Integradas, con expectativas de promover la investigación, creando grupos de trabajo en cada una de sus diferentes centros de actividad educativa.
Con este programa pretendemos lograr una aproximación a lo que sería la enseñanza integrada de las Ciencias Naturales en los niveles de: básica primaria, secundaria y media vocacional, con base en la tendencia en la cual el aprendiz es agente de su propio aprendizaje ("aprender a aprender"). Conscientes de la complejidad del problema y que su solución no es tan simple debido a la diversidad de métodos y técnicas que son utilizadas en cada una de las disciplinas en que se dividen la Ciencia Naturales, consideramos, puede funcionar como un agente impulsor en la unificación del currículo en torno a la enseñanza de las Ciencias Naturales Integradas de nuestra región.

Desde nuestro punto de vista, la manera práctica de integrar las Ciencias Naturales es sobre la base de un currículo desarrollado en torno a problemas y no a disciplinas, y el punto estratégico se encuentra precisamente en la crisis ambiental, generada por la manera como el hombre se relaciona con el medio ambiente.

Este es un punto estratégico porque precisamente la crisis ambiental ha puesto en evidencia la ambivalencia de las organizaciones sociales y de las comunidades científicas. Una de las críticas que viene planteando el ambientalismo al desarrollo de las Ciencias, es la ineficacia de las mismas para el análisis de los sistemas complejos. La dificultad surge por la incapacidad de las diferentes disciplinas de desarrrollar un diálogo académico, debido a la relativa insularidad de cada una de ellas.

Además, al retomar la integración de estas disciplinas en torno al problema ecológico, las instituciones educativas de básica primaria, de básica secundaria y 
media vocacional, estarán dando respuestas a las exigencias de Decreto $1857 \mathrm{del} 3$ de agosto de 1994 que obliga a incluir la educación ambiental en todos los niveles al igual que al decreto 1743 de 1994 que obliga a todas las intituciones educativas a desarrollar un proyecto ambiental dentro del Proyecto Educativo Institucional.

En términos generales, a través del Programa de Especialización en Informática para la Docencia de las Ciencicas Naturales, estamos presentando alter- nativas de solución a los problemas que hemos venido planteando y se se basan en:

1. La inclusión de la educación ambiental como núcleo unificador.

2. El uso de la Informática como herramienta de mejormiento pedagógico.

3. Las nuevas estrategias metodológicas derivadas de la Sicología y la Epistemología basadas en el "aprender a aprender".

\section{BIBLIOGRAFÍA}

1. GONZÁleZ S, Hernando y PERALTA G, Clotario I. Proyecto de Especialización en: Informática para la Docencia de las Ciencias Naturales. Unversidad Surcolombiana, Mayo de 1997.

2. CAICEDO LÓPEZ, Humberto "Una Alternativa en la enseñanza de las Ciencias". En: Revista Ciencia y Tecnología Universidad Pedagógica Nacional, 1991.

3. PEREA E, Juan Manuel "Reseña histórica del programa de Licenciatura en Matemáticas y Física de la Universidad Surcolombiana". Revista de Facultad de Educación de la Universidad Surcolombiana PAIDEIA No. 5 Febrero de 1997. 